\title{
High-Resolution Single-Snapshot DOA Estimation in MIMO Radar with Colocated Antennas
}

\author{
Aboulnasr Hassanien, Moeness G. Amin, Yimin D. Zhang, and Fauzia Ahmad \\ Center for Advanced Communications, Villanova University, Villanova, PA 19085, USA \\ hassanien@ieee.org, \{moeness.amin,yimin.zhang, fauzia.ahmad\}@villanova.edu
}

\begin{abstract}
In this paper, a high-resolution subspace-based method is proposed for single-snapshot direction-of-arrival (DOA) estimation in multiple-input multiple-output (MIMO) radar. First, a 'principal' weight vector is designed for transmit beamforming to achieve a desired transmit power distribution, e.g., to focus the transmit power within certain desired spatial sector(s), while minimizing the out-of-sector power. Then, the designed weight vector is used to generate a population of 'companion' weight vectors of the same size and having the same transmit power distribution as that of the principal weight vector. Multiple transmit beams are formed using the companion weight vectors where an orthogonal waveform is transmitted over each beam. Matched-filtering the received single-snapshot data to the orthogonal transmitted waveforms yields a number of virtual data snapshots equal to the number of orthogonal waveforms. By properly choosing the number of waveforms, the virtual data snapshots are guaranteed to yield a full-rank sample covariance matrix, which enables application of high-resolution DOA estimation techniques. Simulation results demonstrate that the proposed method provides DOA estimation performance superior to that of traditional MIMO radar based MUSIC, the conventional (Bartlett) beamformer, and the spheroidal sequences based beamformer.
\end{abstract}

\section{INTRODUCTION}

Direction-of-arrival (DOA) estimation is a fundamental problem that finds applications in many diverse areas, such as radar, sonar, wireless communications, seismology, radio astronomy, and medical diagnostics [1]. Adopting the terminology in [2], existing DOA estimation techniques can be classified into non-parametric and parametric techniques. The simplest non-parametric DOA estimation technique is the conventional (Bartlett) beamformer. Despite its simplicity, the conventional beamformer suffers from high secondary lobes and low-resolution limit. High-resolution non-parameteric techniques include the Capon beamformer, MUSIC, ESPRIT, and other subspace-based DOA estimation techniques. On the other hand, parametric DOA estimation techniques, such as the deterministic and stochastic maximum likelihood methods, offer excellent DOA estimation performance at the price of high computational cost due to the multidimensional search over the unknown parameters. Most of the existing DOA estimation techniques have been developed for the classical singleinput multiple-output (SIMO) sensor array configurations. The performances of these techniques have been thoroughly investigated based on asymptotic assumptions, and shown to deteriorate when a small number of snapshots is available. Moreover, all high-resolution methods which involve inverting the sample covariance matrix are not applicable unless the covariance matrix is full-rank.

Recently, the problem of DOA estimation using a singlesnapshot has received considerable attention [3]-[4]. It is not uncommon in practice to have situations where there is only a few data snapshots or even a single-snapshot available for DOA estimation. For example, in smart automotive systems, adaptive cruise control and/or emergency brake initiation require performing online estimation of the relative distances and speeds with respect to other targets as well as the target DOAs [3]. Due to the rapid change of the relative ranges, velocities, and DOAs, a single-snapshot could be the only available data for DOA estimation. Another example can be found in sonar applications where the physical limitations associated with the sonar system and the complicated characteristics of the underwater signal channel dramatically reduce the coherent processing interval to a few, if not a single-snapshot. As a result, the sample covariance matrix becomes rank-deficient, rendering DOA estimation using high-resolution techniques infeasible.

In the last decade, the concept of multiple-input multipleoutput (MIMO) radar has been the focus of intensive research [7]-[9]. It has been shown that MIMO radar with colocated transmit/receive antennas enables improved angular resolution and parameter identifiability, increased number of detectable targets, and extended array aperture by virtual sensors. Many algorithms have been developed for DOA estimation in MIMO radar based on a large available number of data snapshots [10]-[12] as well as using a single pulse [10], [13]. However, without employing proper transmit beamforming, MIMO radar suffers from the loss of coherent transmit processing gain. This results in weaker signal strengths at the receive array which, in turn, affect DOA estimation performance.

In this paper, we consider the problem of DOA estimation of multiple targets in MIMO radar using a single snapshot, i.e., using data collected from a single radar pulse. A highresolution subspace-based method for single-snapshot DOA estimation is introduced ${ }^{1}$. We employ transmit beamforming to achieve transmit coherent processing. A transmit weight vector is properly designed to provide a desired transmit power distribution pattern, e.g., to focus the transmit power within certain spatial sector(s), while minimizing the power radiated in the out-of-sector directions. This weight vector is referred to as the 'principal' weight vector, which is used to generate

${ }^{1}$ Related formulations have been reported in [14]. 
a population of weight vectors of the same dimensionality and of the same transmit power distribution as that of the principal weight vector ${ }^{2}$. A subset of the aforementioned weight vectors can be selected and used to simultaneously transmit a set of orthogonal waveforms, i.e., the number of orthogonal transmit waveforms equals the number of weight vectors in the subset. Matched-filtering the single-snapshot received data to the orthogonal transmit waveforms yields a number of virtual data snapshots equals to the number of orthogonal waveforms. In so doing, a full-rank sample covariance matrix can be constructed using the virtual snapshots, provided the number of orthogonal waveforms is properly chosen. This enables the application of high-resolution techniques for DOA estimation. We use simulation examples to validate the effectiveness of the proposed single-snapshot DOA estimation method.

\section{SYSTEM MODEL}

We assume a MIMO radar system equipped with $M$ transmit and $N$ receive colocated antennas. The transmit antennas are assumed to be arranged in a uniform linear array (ULA) while the receive antenna array can be of an arbitrary shape. Note that the formulations derived hereafter can be applied in a straightforward manner to sparse symmetric transmit arrays. However, in this paper, we focus on ULAs at the transmitter for the sake of simplicity.

Let $\mathbf{w}$ be the $M \times 1$ principal transmit beamforming weight vector. The main function of $\mathbf{w}$ is to concentrate the transmit power within a certain desired spatial sector $\boldsymbol{\Theta}=\left[\theta_{\min }, \theta_{\max }\right]$ (or multiple sectors) while minimizing the power radiated in the out-of-sector region $\overline{\boldsymbol{\Theta}}$. There are several methods in the literature which can be used for transmit beamforming design (see for example [16] and references therein). Here, we design $\mathrm{w}$ by solving the following optimization problem

$$
\begin{aligned}
& \min _{\mathbf{w}} \max _{i}\left|\mathbf{w}^{H} \mathbf{a}\left(\theta_{i}\right)-e^{j \phi\left(\theta_{i}\right)}\right|, \quad \theta_{i} \in \mathbf{\Theta}, i=1, \ldots, I \\
& \text { subject to }\left|\mathbf{w}^{H} \mathbf{a}\left(\theta_{k}\right)\right| \leq \delta, \quad \theta_{k} \in \overline{\boldsymbol{\Theta}}, k=1, \ldots, K,
\end{aligned}
$$

where $\mathbf{a}(\theta)$ is the transmit steering vector, $\theta_{i}$ and $\theta_{k}$ are continuums of angles chosen uniformly or non-uniformly to approximate $\boldsymbol{\Theta}$ and $\boldsymbol{\Theta}$, respectively, $\phi(\theta)$ is an arbitrary phase profile which is assumed to be continuous within the desired sector $\boldsymbol{\Theta}, \delta$ is a user-specified positive number to control sidelobe levels, and $(\cdot)^{H}$ denotes the Hermitian operator. The optimization problem (1) is convex and can be efficiently solved using the interior point methods [17].

Let $\mathbf{w}_{q}, q=1, \ldots, Q$, be a set of companion weight vectors of dimension $M \times 1$ each. The companion weight vectors can be generated from the principal weight $\mathbf{w}$ using the method detailed in [15]. Without loss of generality, we assume that each companion weight vector is normalized to have unit norm. Each companion weight vector has the same transmit power distribution pattern as the principal weight vector, i.e.,

$$
\left|\mathbf{w}^{H} \mathbf{a}(\theta)\right|^{2}=\left|\mathbf{w}_{q}^{H} \mathbf{a}(\theta)\right|^{2}, \quad \theta \in\left[\frac{-\pi}{2}, \frac{\pi}{2}\right], q=1, \ldots, Q .
$$

Let $\boldsymbol{\Psi}(t)=\left[\psi_{1}(t), \ldots, \psi_{Q}(t)\right]^{T}$ be the $Q \times 1$ vector of transmit waveforms where $\left\{\psi_{q}(t)\right\}, q=1, \ldots, Q$, is a predesigned set

\footnotetext{
${ }^{2}$ More information on how to generate a population of companion weight vectors from a principal weight vector can be found in [15].
}

of orthogonal waveforms, $t$ is the fast time, and $(\cdot)^{T}$ denotes matrix transpose. Each companion weight vector is used to form a transmit beam for radiating one of the orthogonal waveforms. Therefore, the baseband representation of the $M \times 1$ transmit signal vector $\mathbf{x}(t)$ can be expressed as a linear combination of the individual orthogonal waveforms, i.e.,

$$
\mathbf{x}(t)=\eta \mathbf{W} \mathbf{\Psi}(t)
$$

where $\mathbf{W}=\left[\mathbf{w}_{1}^{*}, \ldots, \mathbf{w}_{Q}^{*}\right]$ is the $M \times Q$ transmit beamforming weight matrix, $\eta=\sqrt{M / Q}$ is a normalization factor used to ensure that the total transmit power is fixed to $P_{t}=M$, and $(\cdot)^{*}$ denotes complex conjugate. Note that $\mathbf{x}(t)$ is the vector of the baseband signals used at the transmit side of the MIMO radar system. From a practical viewpoint, transmit power efficiency is achieved employing constant modulus signals. It is noted, however, that from a receiver perspective, the signal model (3) enables performing pulse compression using the signal vector $\boldsymbol{\Psi}(t)$ which need not be constant modulus. This permits additional degrees of freedom in designing the individual orthogonal waveforms.

The baseband representation of the $N \times 1$ received signal vector at the output of the receive array due to a hypothetical target in direction $\theta$ is modeled as

$$
\begin{aligned}
\mathbf{y}(t) & =\eta \beta_{\theta} \mathbf{b}(\theta) \mathbf{a}^{T}(\theta) \mathbf{x}(t)+\mathbf{n}(t) \\
& =\eta \beta_{\theta} \mathbf{b}(\theta) \mathbf{a}^{T}(\theta) \mathbf{W} \mathbf{\Psi}(t)+\mathbf{n}(t),
\end{aligned}
$$

where $\beta_{\theta}$ is the target reflection coefficient, $\mathbf{b}(\theta)$ is the $N \times 1$ receive steering vector, $\mathbf{n}(t)$ is the $N \times 1$ vector of additive white Gaussian noise with zero mean and covariance $\sigma_{z}^{2} \mathbf{I}_{N}$, and $\mathbf{I}_{N}$ denotes the $N \times N$ identity matrix. In (4), the reflection coefficient $\beta_{\theta}$ is assumed to obey the Swirlling II target model, i.e., it remains constant during the entire width of the radar pulse.

\section{High-RESOLUTION DOA ESTIMATION}

Assuming that $L$ targets co-exist in a certain range bin, matched-filtering the received data (4) to each of the orthogonal transmitted waveforms yields the virtual data snapshots

$$
\mathbf{y}_{q}=\eta \sum_{l=1}^{L} \beta_{l}\left(\mathbf{w}_{q}^{H} \mathbf{a}\left(\theta_{l}\right)\right) \mathbf{b}\left(\theta_{l}\right)+\mathbf{n}_{q}, \quad q=1, \ldots, Q,
$$

where $\beta_{l}$ is the reflection coefficient associated with the $l$ th target, $\mathbf{n}_{q}=\int_{\mathrm{Tp}} \mathbf{n}(t) \psi_{q}^{*}(t) d t$ is the $N \times 1$ noise vector at the output of the matched filter which has the same statistics as that of $\mathbf{n}(t)$, and $\mathrm{Tp}$ is the radar pulse duration.

Utilizing the virtual data snapshots (5), the $N \times N$ sample covariance matrix can be computed as

$$
\hat{\mathbf{R}}=\frac{1}{Q} \sum_{q=1}^{Q} \mathbf{y}_{q} \mathbf{y}_{q}^{H}
$$

Assume that the number of transmit antennas is larger than or equal to the number of receive antennas, i.e., $M \geq N$. Then, by choosing $Q \geq M$, the sample covariance matrix (6) is guaranteed to be full-rank. It is worth noting that for the case when $N>M$, the virtual data at the output of the matched-filters can be re-arranged such that a full-rank sample 
covariance matrix of size $M \times M$ can be constructed. However, we do not consider this case here for the sake of brevity.

Performing eigendecomposition of (6), we obtain the $N \times L$ matrix $\mathbf{U}_{s}$ and $N \times(N-L)$ matrix $\mathbf{U}_{n}$, which correspond to the signal subspace and noise subspace, respectively. The MUSIC spectrum can then be computed as

$$
P(\theta)=\frac{\mathbf{b}^{H}(\theta) \mathbf{b}(\theta)}{\mathbf{b}^{H}(\theta) \mathbf{U}_{n} \mathbf{U}_{n}^{H} \mathbf{b}(\theta)} .
$$

The DOAs are obtained by searching for the $L$ largest peaks in the MUSIC spectrum (7).

\section{COMparison to Existing Methods}

In this section, we draw comparison between the proposed formulations and three existing methods, namely, the traditional MIMO radar based MUSIC, the conventional (Bartlett) beamformer, and the spheroidal sequences based DOA estimation, which can be applied to the single-snapshot case. In particular, we show that each of the three aforementioned methods can be viewed as a special case of our proposed formulations.

\section{A. Traditional MIMO Radar Configuration}

Traditional MIMO radar is based on transmitting $Q=M$ orthogonal waveforms, one from each transmit antenna. This corresponds to choosing the transmit beamforming weight matrix to be the $M \times M$ identity matrix, i.e., $\mathbf{W}=\mathbf{I}_{M}$. In this case, the virtual snapshots in (5) reduce to

$$
\mathbf{y}_{m}=\sum_{l=1}^{L} \beta_{l} \mathbf{a}_{[m]}\left(\theta_{l}\right) \mathbf{b}\left(\theta_{l}\right)+\mathbf{n}_{m}, \quad m=1, \ldots, M,
$$

where $(\cdot)_{[m]}$ is the $m$ th entry of a vector. It is worth noting that in (8), the power normalization factor $\eta$ equals unity and, therefore, is omitted from the equation. A full-rank covariance matrix can be constructed using the virtual snapshots (8) which enables application of MUSIC for DOA estimation. However, it can be observed from (8) that the traditional MIMO radar suffers from decreased signal strength due to the fact that no coherent processing gain is used on transmit.

\section{B. Classical SIMO Radar Configuration}

In the classical SIMO radar configuration, a single transmit waveform is employed. Transmit coherent processing gain is achieved using a single transmit beamforming weight vector. This case corresponds to choosing the transmit weight matrix $\mathbf{W}$ in the proposed formulation to be a single vector, e.g., the principal weight vector. In such a case, the virtual snapshots (5) reduce to a single data snapshot

$$
\mathbf{y}_{\text {SIMO }}=\sqrt{M} \sum_{l=1}^{L} \beta_{l}\left(\mathbf{w}^{H} \mathbf{a}\left(\theta_{l}\right)\right) \mathbf{b}\left(\theta_{l}\right)+\mathbf{n} .
$$

By comparing (5) and (9), it is observed that both formulations have the same power distribution pattern. However, the SIMO configuration has higher signal strength at the receiver as a result of assigning the total transmit power to a single waveform. Despite the high signal strength, the single-snapshot data does not enable high-resolution DOA estimation. Therefore, the conventional (Bartlett) beamformer has to be used for DOA estimation. The DOAs can be obtained by searching for the $L$ highest peaks in the conventional beamformer spectrum, i.e.,

$$
P_{\mathrm{CB}}(\theta)=\frac{\left|\mathbf{b}^{H}(\theta) \mathbf{y}_{\mathrm{SIMO}}\right|^{2}}{\mathbf{b}^{H}(\theta) \mathbf{b}(\theta)} .
$$

\section{Spheroidal Sequences Based Configuration}

The discrete prolate spheroidal sequences can be used to incorporate coherent processing gain at the transmit side of the MIMO radar system. This corresponds to choosing the transmit beamforming weight matrix as $\mathbf{W}=\left[\mathbf{u}_{1}, \ldots, \mathbf{u}_{K}\right]$, where $\mathbf{u}_{k}, k=1, \ldots, K$, are the $K(K<M)$ principal eigenvectors of the positive semi-definite matrix $\mathbf{A}=\int_{\Theta} \mathbf{a}(\theta) \mathbf{a}^{H}(\theta) d \theta$. In this case, the virtual snapshots given in $(5)$ reduce to

$$
\mathbf{y}_{k}=\eta_{s} \sum_{l=1}^{L} \beta_{l}\left(\mathbf{u}_{k}^{H} \mathbf{a}\left(\theta_{l}\right)\right) \mathbf{b}\left(\theta_{l}\right)+\mathbf{n}_{k}, \quad k=1, \ldots, K,
$$

where $\eta_{s}=\sqrt{M / K}$. Since $K$ is small, the $K$ virtual snapshots (11), in general, do not yield a full-rank sample covariance matrix. Similar to the SIMO case, a low-resolution DOA estimation can be performed by searching for the $L$ highest peaks of the spectrum

$$
P_{\mathrm{SPH}}(\theta)=\frac{\mathbf{b}^{H}(\theta) \hat{\mathbf{R}}_{\mathrm{SPH}} \mathbf{b}^{H}(\theta)}{\mathbf{b}^{H}(\theta) \mathbf{b}(\theta)},
$$

where $\hat{\mathbf{R}}_{\mathrm{SPH}}=\sum_{k=1}^{K} \mathbf{y}_{k} \mathbf{y}_{k}^{H}$.

\section{Simulation Results}

The MIMO radar is assumed to have a transmit ULA with $M=12$ antennas spaced half-wavelength apart, and a non-uniform linear receive array of $N=10$ elements. The locations of the receive antennas are randomly drawn from the range $\left[\begin{array}{ll}0 & 9\end{array}\right]$ measured in units of half-wavelengths. The additive noise signals are modeled as Gaussian, zero-mean, and both temporally and spatially white. The targets are assumed to be located within the spatial sector $\Theta=\left[-10^{\circ}, 10^{\circ}\right]$. The principal weight vector is designed by solving (1) where the phase profile $\phi(\theta)=2 \pi \sin \theta$ is chosen. The sidelobe levels are controlled by choosing $\delta=\sqrt{0.01}$, i.e., the sidelobe attenuation is at least $20 \mathrm{~dB}$. Following the guidelines of [15], the principal weight vector obtained by solving (1) is used to generate a population of 4095 companion weight vectors. The principal weight vector in addition to the 4095 companion weight vectors form together an overall population of 4096 weight vectors. $Q=12$ weight vectors are drawn randomly from the overall population and used for the proposed singlesnapshot DOA estimation. For the conventional beamformer, only the principal weight vector is used at the transmit side. For the spheroidal sequences based method, $K=3$ principal eigenvectors are used. For all methods tested, the total transmit power is fixed to $P_{t}=M$.

Fig. 1 shows the normalized transmit power distribution for the proposed method and the spheroidal sequences based method. For the traditional MIMO configuration, the transmit power distribution is flat due to the onmidirectional transmission of the waveforms and, therefore, is not shown in the figure. For the classical SIMO configuration, the transmit 


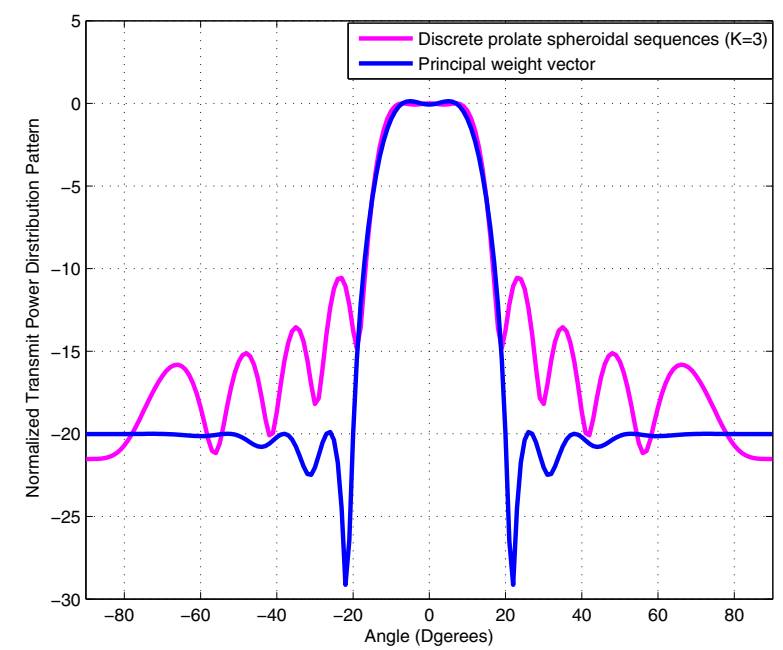

Fig. 1. Transmit power distribution versus spatial angle.

power distribution is the same as that of the proposed method because the employed transmit weight vector is the principal weight vector designed for the proposed scheme. It can be observed from the figure that the transmit power distribution of the spheroidal sequences based method has higher sidelobe levels as compared to the proposed method.

To test the high resolution capability of the proposed method, two targets are assumed to be located at $-2^{\circ}$ and $2^{\circ}$. Fig. 2 depicts the root-mean square error (RMSE) versus the signal-to-noise ratio (SNR) for all considered methods. The results are averaged over 300 independent runs in each case. We observe that both conventional and spheroidal sequences beamformers have poor performance at moderate and high SNR values, which can be attributed to the low-resolution and biased features of the estimators. The figure also shows that the traditional MIMO based MUSIC has good RMSE performance especially at high SNR values. Finally, it can be seen from the figure that the proposed single-snapshot MUSIC has the best RMSE performance as compared to all other methods.

Fig. 3 plots the probability of target resolution versus the SNR for all considered methods. The targets are assumed to be resolved if at least two peaks in the spectrum are observed and the following condition is satisfied [1]

$$
\left|\hat{\theta}_{l}-\theta_{l}\right| \leq \frac{\left|\theta_{2}-\theta_{1}\right|}{2} \quad l=1,2 .
$$

It can be observed from Fig. 3 that the conventional beamformer and the spheroidal sequences based method cannot achieve a good probability of target resolution performance even at very high SNR values mainly due to the low-resolution capability of the two estimators. The proposed single-snapshot MUSIC and the traditional MIMO based MUSIC achieve a $100 \%$ resolution for SNR values higher than $5 \mathrm{~dB}$ and $8 \mathrm{~dB}$, respectively. For smaller SNR values, the proposed method provides better resolution capabilities as compared to the traditional MIMO based MUSIC. It is worth noting that the target separation used in this example is $\Delta \theta=\theta_{2}-\theta_{1}=4^{\circ}$ which is smaller than the Rayleigh resolution limit. As a result,

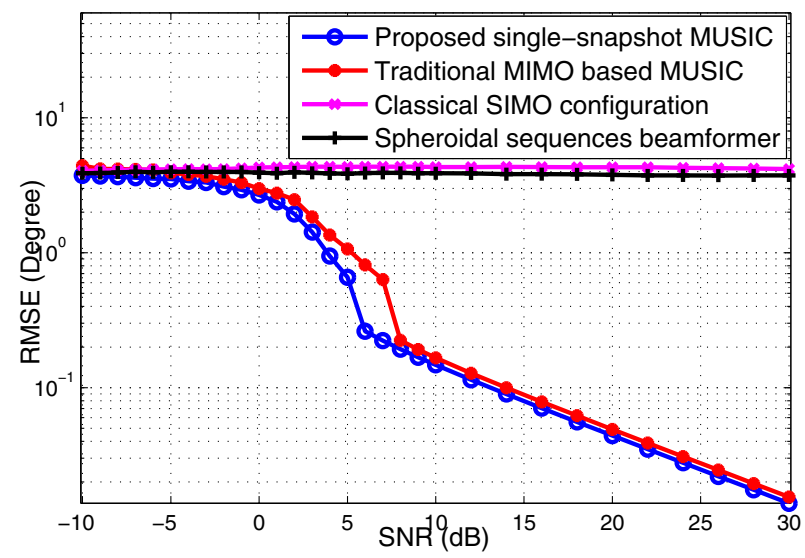

Fig. 2. RMSE versus SNR.

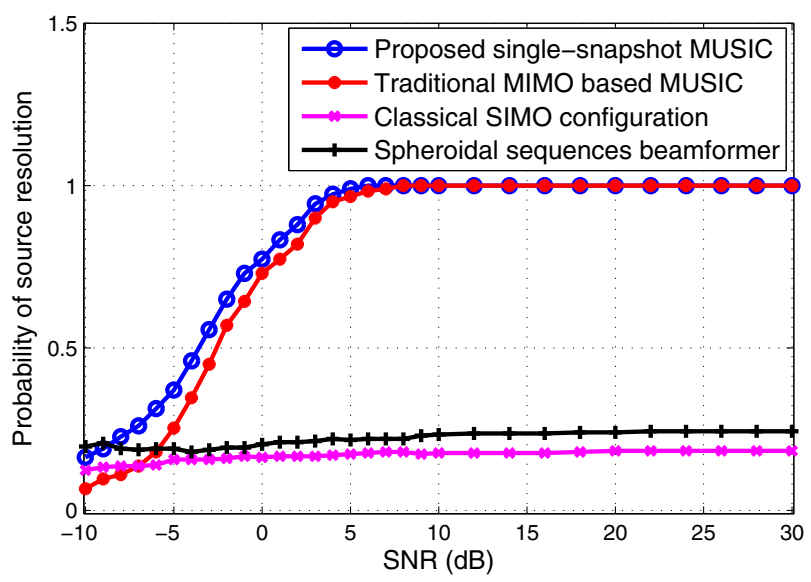

Fig. 3. Probability of target resolution versus SNR.

the conventional and spheroidal sequences beamformers fail to resolve the targets at all SNR values.

\section{CONCLUSION}

The problem of DOA estimation of multiple targets in MIMO radar using a single snapshot has been addressed. A subspace-based method for single-snapshot DOA estimation was proposed. Transmit coherent processing gain was achieved by properly designing a principal transmit weight vector to focus the transmit power within desired spatial sector(s) while minimizing the power radiated in the out-of-sector directions. The designed weight vector was used to generate a population of companion weight vectors with same transmit power distribution as that of the principal vector. The companion weight vectors enable transmitting multiple orthogonal waveforms over a single illumination, which lend themselves to providing a number of virtual data snapshots equal to the number of orthogonal waveforms, thereby permitting the application of high-resolution DOA estimation techniques. The proposed method was shown to outperform the classical MIMO radar, the conventional (Bartlett) beamformer, and the spheroidal sequences based beamformer. 


\section{REFERENCES}

[1] H. L. Van Trees, Optimum Array Processing. Wiley, NY, 2002.

[2] H. Krim and M. Viberg, "Two decades of array signal processing research: the parametric approach," IEEE Signal Processing Mag., vol. 13, no. 4, pp. 67-94, Aug. 1996.

[3] P. Häcker and B. Yang, "Single snapshot DOA estimation," Adv. Radio Sci. 8, no. 16, pp. 251-256, 2010.

[4] P. Heidenreich and A. M. Zoubir, "Computationally simple DOA estimation of two resolved targets with a single snapshot," in Proc. 37th IEEE Int. Conf. Acoustics, Speech and Signal Processing (ICASSP'12), Kyoto, Japan, Mach 2012, pp. 2553-2556.

[5] S. Fortunati, R. Grasso, F. Gini, and M. S. Greco, "Single snapshot DOA estimation using compressed sensing," in Proc. 39th IEEE Int. Conf. Acoustics, Speech and Signal Processing (ICASSP'14), Florence, Italy, May 2014, pp. 2297-2301.

[6] J. Li and P. Stoica, "MIMO radar with colocated antennas," IEEE Signal Processing Mag., vol. 24, pp. 106-114, Sept. 2007.

[7] J. Li and P. Stoica, MIMO Radar Signal Processing. New Jersy: Wiley, 2009.

[8] A. Hassanien and S. A. Vorobyov, "Phased-MIMO radar: A tradeoff between phased-array and MIMO radars," IEEE Trans. Signal Processing, vol. 58, no. 6, pp. 3137-3151, June 2010.

[9] A. Hassanien and S. A. Vorobyov, "Transmit energy focusing for DOA estimation in MIMO radar with colocated antennas," IEEE Trans. Signal Processing, vol. 59, no. 6, pp. 2669-2682, June 2011.
[10] D. Nion and N. D. Sidiropoulos, "Tensor algebra and multidimensional harmonic retrieval in signal processing for MIMO radar," IEEE Trans. Signal Processing, vol. 58, no. 11, pp. 5693-5705, Nov. 2010.

[11] Y. D. Zhang, M. G. Amin, and B. Himed, "Joint DOD/DOA estimation in MIMO radar exploiting time-frequency signal representations," EURASIP Journal on Advances in Signal Processing, vol. 2012, no. 1, July 2012.

[12] A. Khabbazibasmenj, A. Hassanien, S. Vorobyov, and M. Morrency, "Efficient transmit beamspace design for search-free based DOA estimation in MIMO radar," IEEE Trans. Signal Processing, vol. 62, no. 6, pp. 1490-1500, Mar. 2014.

[13] L. Xu, J. Li, and P. Stoica, "Target detection and parameter estimation for MIMO radar systems," IEEE Trans. Aerosp. Electron. Syst., vol. 44, no. 3, pp. 927-939, July 2008.

[14] A. Hassanien, M. G. Amin, Y. D. Zhang, and F. Ahmad, "Capon-based single snapshot DOA estimation in monostatic MIMO Radar," in Proc. Symposium SPIE Sensing Technology + Applications, Baltimore, MD, Apr. 2015.

[15] A. Khabbazibasmenj, A. Hassanien, and S. A. Vorobyov, "How many beamforming vectors generate the same beampattern?," IEEE Signal Processing Lett., submitted, arXiv preprint, arXiv:1402.1682, 2014.

[16] G. Hua and S. S. Abeysekera, "MIMO radar transmit beampattern design with ripple and transition band control," IEEE Trans. Signal Processing, vol. 61, no. 11, pp. 2963-2974, Jun. 2013

[17] S. Boyd and L. Vandenberghe, Convex optimization. Cambridge university press, 2009. 